INPLASY

PROTOCOL

To cite: Tang et al. Network Meta-analysis of Heat-clearing and Detoxifying Oral Liquid of Chinese Medicines in Treatment of Children's Hand-foot-mouth

Disease: a protocol for systematic review. Inplasy protocol 202210032. doi: 10.37766/inplasy2022.1.0032

Received: 07 January 2022

Published: 07 January 2022

Corresponding author: Jiqin Tang

tangjiqin0312@163.com

Author Affiliation:

Shandong University of Traditional Chinese Medicine.

Support: Shandong University of TCM.

Review Stage at time of this submission: Preliminary searches.

Conflicts of interest: None declared.

\section{Network Meta-analysis of Heat-clearing and Detoxifying Oral Liquid of Chinese Medicines in Treatment of Children's Hand-foot-mouth Disease: a protocol for systematic review}

\author{
Tang, J1; Zhang, G2; Xing, X3; Yu, Y4; Han, T5.
}

Condition being studied: Hand foot mouth disease (HFMD) is a common infectious disease in pediatrics caused by a variety of enteroviruses. Its clinical manifestations are mainly characterized by persistent fever, hand foot rash, oral herpes, ulcers, etc. Because it is often found in preschool children, its immune system development is not perfect, so it is very vulnerable to infection by pathogens and epidemic diseases, resulting in rapid progress of the disease. A few patients will also have neurogenic pulmonary edema Meningitis, myocarditis and other serious complications even lead to death, so effectively improve the cure rate, shorten the course of disease, prevent the deterioration of the disease as the focus of the study. In recent years, traditional Chinese medicine has played an important role in the research of antiviral treatment. Many clinical practices have confirmed that oral liquid of traditional Chinese medicine can effectively play the role of antiviral and improve the body's immunity.

INPLASY registration number: This protocol was registered with the International Platform of Registered Systematic Review and Meta-Analysis Protocols (INPLASY) on 07 January 2022 and was last updated on 07 January 2022 (registration number INPLASY202210032).

\section{INTRODUCTION}

Review question / Objective: The type of study was clinical randomized controlled trial (RCT). The object of study is the patients with HFMD. There is no limit to gender and race. In the case of clear diagnosis standard, curative effect judgment standard and consistent baseline treatment, the experimental group was treated with pure oral liquid of traditional Chinese medicine(A: Fuganlin oral liquid, B: huangzhihua oral liquid, C: Lanqin oral liquid, D: antiviral oral liquid, E: Huangqin 
oral liquid, F: Pudilan oral liquid, G: Shuanghuanglian oral liquid.) and the control group was treated with ribavirin or any oral liquid of traditional Chinese medicine. The data were extracted by two researchers independently, cross checked and reviewed according to the predetermined tables. The data extraction content is (1) Basic information (including the first author, published journal and year, research topic). (2) Relevant information (including number of cases, total number of cases, gender, age, intervention measures, course of treatment of the experimental group and the control group in the literature). (3) Design type and quality evaluation information of the included literature. (4) Outcome measures (effective rate, healing time of oral ulcer, regression time of hand and foot rash, regression time of fever, adverse reactions.). The seven traditional Chinese medicine oral liquids are comparable in clinical practice, but their actual clinical efficacy is lack of evidence-based basis. Therefore, the purpose of this study is to use the network meta-analysis method to integrate the clinical relevant evidence of direct and indirect comparative relationship, to make quantitative comprehensive statistical analysis and sequencing of different oral liquid of traditional Chinese medicine with the same evidence body for the treatment of the disease, and then to explore the advantages and disadvantages of the efficacy and safety of different oral liquid of traditional Chinese medicine to get the best treatment plan, so as to provide reference value and evidence-based medicine evidence for clinical optimization of drug selection.

Rationale: Traditional Chinese medicine, as an important part of complementary and alternative medicine, has played a huge advantage in the research of antiviral treatment. Many clinical practices have confirmed that a variety of oral liquid of Chinese patent medicine developed from natural herbs has been widely used in the treatment of children's viral hand-footmouth disease. This study is to use the network meta-analysis method to integrate the clinical relevant evidence of direct and indirect comparative relationship, to make quantitative comprehensive statistical analysis and sequencing of different oral liquid of traditional Chinese medicine with the same evidence body for the treatment of the disease, and then to explore the advantages and disadvantages of the efficacy and safety of different oral liquid of traditional Chinese medicine to get the best treatment plan, so as to provide reference value and evidence-based medicine evidence for clinical optimization of drug selection.

Condition being studied: Hand foot mouth disease (HFMD) is a common infectious disease in pediatrics caused by a variety of enteroviruses. Its clinical manifestations are mainly characterized by persistent fever, hand foot rash, oral herpes, ulcers, etc. Because it is often found in preschool children, its immune system development is not perfect, so it is very vulnerable to infection by pathogens and epidemic diseases, resulting in rapid progress of the disease. A few patients will also have neurogenic pulmonary edema Meningitis, myocarditis and other serious complications even lead to death, so effectively improve the cure rate, shorten the course of disease, prevent the deterioration of the disease as the focus of the study. In recent years, traditional Chinese medicine has played an important role in the research of antiviral treatment. Many clinical practices have confirmed that oral liquid of traditional Chinese medicine can effectively play the role of antiviral and improve the body's immunity.

\section{METHODS}

Search strategy: By using the computer retrieval technology, a comprehensive search was carried out for the relevant literature of the randomized controlled trial of the clinical efficacy and safety of seven kinds of oral liquid of traditional Chinese medicine in the treatment of children's hand, foot and mouth disease, and the self built database was set up until October 31, 2021. Computer retrieval includes CNKI (Chinese journal full text database), CBM (Chinese biomedical literature database), 
Wanfang Data (Wanfang science and technology information database), VIP (China Weipu science and technology journal database) and other Chinese databases. The Cochrane Library, PubMed, Embase and Web of Science and other foreign databases were also searched, and relevant literature was consulted and supplemented in the reference of clinical trial reports or reviews. Chinese search terms are mainly oral liquid, hand foot mouth, ribavirin, random control, etc. And different databases choose corresponding free words and subject word combinations, english search terms are oral liquid, ribavirin, hand foot and mouth disease, randomized controlled trials.

Participant or population: The object of study is children with severe HFMD. All cases must conform to the diagnosis standard of HFMD issued by the ministry of health of China, which has the following characteristics: epidemic characteristics, fever, hand foot and palm rash, some cases occur in the buttocks, oral herpes and HFMD are at the same time or prior to HFMD, it may be accompanied by cough, salivation, anorexia, vomiting and other manifestations, regardless of gender and race. In the case of clear diagnosis standard, curative effect judgment standard and consistent baseline treatment, the experimental group was treated with pure oral liquid of traditional Chinese medicine, and the control group was treated with ribavirin or any oral liquid of traditional Chinese medicine.

Intervention: In the case of clear diagnosis standard, curative effect judgment standard and consistent baseline treatment, the experimental group was treated with pure oral liquid of traditional Chinese medicine(A: Fuganlin oral liquid, B: huangzhihua oral liquid, C: Lanqin oral liquid, D: antiviral oral liquid, E: Huangqin oral liquid, F: Pudilan oral liquid, G: Shuanghuanglian oral liquid.)and the control group was treated with ribavirin or any of the above six kinds of oral liquid of traditional Chinese Medicine.
Comparator: The control group was treated with ribavirin or any oral liquid of traditional Chinese medicine(A: Fuganlin oral liquid, B: huangzhihua oral liquid, C: Lanqin oral liquid, D: antiviral oral liquid, E: Huangqin oral liquid, F: Pudilan oral liquid, G: Shuanghuanglian oral liquid.).

Study designs to be included: Comprehensive retrieval of CNKI, VIP, CBM and WANFANG database and the Cochrane Library, PubMed, Web of Science and EMBASE database. The type of study was clinical randomized controlled trial (RCT), and there were no restrictions on the sex and race of these patients.Data merging and network meta-analysis were carried out with $R$ programming software to evaluate the ranking probability of all interventions.

Eligibility criteria: The design of inclusion criteria and exclusion criteria in this study is based on the five main principles of PICOS. The patient is a single viral HFMD child patient, whose gender, race and region are not limited. The diagnostic criteria of HFMD used shall conform to one of the following: guidelines for diagnosis and treatment of HFMD (2010 Edition) or Zhu Futang Practical Pediatrics (Seventh Edition) and diagnostic efficacy criteria of TCM disease syndromes, and the diagnosis of TCM syndrome type shall conform to the criteria of HFMD in the seventh edition of Practical Pediatrics or the second edition of Pediatrics of TCM. When there are clear diagnostic criteria, curative effect judgment criteria and basic treatment are consistent, one of the Chinese patent medicine oral liquid (Pudilan oral liquid or Lanqin oral liquid or Huangqin oral liquid or Shuanghuanglian oral liquid or Huangzhihua oral liquid or Anti-virus oral liquid or Fuganlin oral liquid) is used as the intervention measure of the experimental group. In the control group, ribavirin alone or one of the seven kinds of oral liquid of Chinese patent medicine was selected as the intervention measures, and no other combination drugs were used.The evaluation indexes are mainly divided into the main outcome indexes and the secondary outcome indexes, among which 
the main outcome indexes include: Clinical total effective rate, Cure rate and Adverse reactions. The secondary outcome indexes include: Healing time of oral ulcer, Disappearance time of hand and foot rash, Hospitalization time, Antipyretic time and other related disease symptom score comparison information acquisition. The literature included were Randomized Controlled Trials (RCTs) with no limitation on language and blind or assignment concealment. As long as the Chinese trial is approved by the local institutional review committee and registered in the international database, we will include its research into the scope of the study. In addition, the author will delete nonrandomized controlled trials, case reports, experience summaries, self-control, and comprehensive literature; animal experimental studies; simple descriptive literature, repeatedly published literature, literature with unclear diagnosis of hand, foot and mouth disease in children or other diseases, the efficacy judgment criteria of the trial group and the control group are not clear, the baseline treatment measures are inconsistent, and the treatment measures are not clear Literature related to the causal relationship interpretation of other treatment methods affecting the final treatment, literature with unclear research results and incomplete data.

Information sources: By using the computer retrieval technology, the literature retrieval of the clinical randomized controlled study on the treatment of children's viral hand, foot and mouth disease with seven kinds of Chinese patent medicine oral liquid was carried out. The primary search was selected and the set period was from the establishment of the database to October 31st, 2021. The computer retrieval electronic database included CNKI, CBM, WANFANG data, VIP and other Chinese databases, as well as the Cochrane Library, PubMed, Web of Science and EMBASE and other foreign databases. Chinese search words include: hand-foot-mouth disease, Chinese patent medicine, Pudilan oral liquid, Lanqin oral I iquid, Huangqin oral liquid, Shuanghuanglian oral liquid, huangzhihua oral liquid, antiviral oral liquid, Fuganlin oral liquid, ribavirin, random, etc. English search words include: (Hand-foot-mouth OR Hand, Foot and Mouth Disease OR Hand, Foot, Mouth Disease OR HFMD) and (Pudilan oral liquid OR Lanqin oral liquid OR Huangqin oral Iiquid OR Shuanghuanglian oral liquid OR Huangzhihua oral liquid OR Antiviral oral liquid OR Fuganlin oral liquid) and (Ribavirin) and (Random*OR randomized controlled trials OR clinical randomized controlled trials).

Main outcome(s): The evaluation indexes are mainly divided into the main outcome indexes and the secondary outcome indexes, among which the main outcome indexes include: Clinical total effective rate, Cure rate and Adverse reactions.

Additional outcome(s): The secondary outcome indexes include: Healing time of oral ulcer, Disappearance time of hand and foot rash, Hospitalization time, Antipyretic time and other related disease symptom score comparison information acquisition.

Data management: According to the retrieval strategy of the above electronic databases, two researchers searched the electronic databases in Chinese and English, used Endnote $X 9$ software to search the repeated information, combined the literature retrieval results in different databases, established the information database and downloaded the full text. Then two first authors independently extract the data for preliminary screening, extract the data according to the predetermined table, take cross check and review, and mark down the reasons for each excluded study, invite the third review researcher to jointly discuss and make a final decision on the research with different opinions. Data extraction content includes 1.Basic information of the included literature (including the first author, published journal and year, research topic). 2.Relevant information of the treatment group and the control group in the literature (including the number of cases, total cases, age, intervention measures, course of treatment, outcome indicators). 
3.Design type and quality evaluation information of the included literature. 4. Outcomes(The main outcome indexes include: Clinical total effective rate, Cure rate and Adverse reactions. The secondary outcome indexes include: Healing time of oral ulcer, Disappearance time of hand and foot rash, Hospitalization time, Antipyretic time and other related disease symptom score comparison information acquisition).

Quality assessment / Risk of bias analysis: According to Cochrane evaluator system recommended by the manual version 5.1 quality evaluation tools, evaluate the methodological quality included in the study by random method, allocation concealment and blinding, ending data integrity, selective reports, fall off the number of cases, follow-up and other bias, and each project is divided into high risk, low risk and uncertainty risk three options, according to the included in the description of the research on the above aspects, the identification and quality evaluation.

Strategy of data synthesis: Revman software is provided by Cochrane collaborative network for literature quality and bias risk assessment. $R$ language programming software is used for direct and indirect results comparison and 95\% $\mathrm{Cl}$ calculation in network meta-analysis. At the same time, network relationship diagram and anecdotal sequence diagram of various interventions are drawn, which can effectively show the indirect comparison relationship of seven kinds of proprietary Chinese medicine oral liquids. The network diagram is mainly composed of nodes and Line composition, in which node represents a treatment mode, the node connected by a straight line represents a direct or indirect comparative relationship between the two, and the thickness of the connecting line represents the number of studies. Then, we will analyze the results of all direct or indirect comparisons to evaluate which is the best treatment plan for children with viral HFMD treated by these seven kinds of Chinese patent medicine oral liquid, and estimate the rank probability of each group based on Markov chain Monte Carlo method. R programming language starts 'NETMETA' program, and calls Bayes MCMC algorithm to analyze the data of random effect model. The OR value (Odd Ratio) was used as the statistical value of efficacy analysis, the weighted mean difference or standardized mean difference (MD) was used as the measurement data, and $95 \% \mathrm{Cl}$ was used for each effect. Odd Ratio (OR) was used as the statistical measure of effective rate, healing time of related symptoms and adverse reactions, and $95 \% \mathrm{Cl}$ confidence intervals was used to express the effect. Based on the network meta probability ranking, in the main outcome indicators, the larger the probability P-value of clinical total effective rate and cure rate, the better the probability P-value of adverse reaction rate. In the information of symptom score comparison of related diseases in secondary outcome indicators, the smaller the P-value is, the better the healing time of oral ulcer, the time of resolving rash of hands and feet, the time of antipyretic, the time of hospitalization, etc.

Subgroup analysis: If significant heterogeneity is found, subgroup analysis will be envisaged based on treatment time, age, race, gender and quality of study to investigate possible sources of heterogeneity.

Sensitivity analysis: If the result of metaanalysis is positive and there are more than 3 included studies, $\mathbf{R}$ software shall be used to conduct sensitivity analysis on the statistical results, and meta-analysis shall be carried out again for each excluded study, and the results shall be compared with those before exclusion. If there is no substantial change in the comparative analysis, the results are stable. Otherwise, the data results are not stable. If significant heterogeneity is found, subgroup analysis will be envisaged based on treatment time, age, race, gender and quality of study to investigate possible sources of heterogeneity.

Language: English.

Country(ies) involved: China. 
Keywords: Chinese patent medicine oral liquid, Ribavirin, Hand-foot-mouth Disease, Network meta-analysis, Protocol.

Contributions of each author:

Author 1 - Jiqin Tang - Author 1 is Jiqin Tang, who is drafted the conceptualization, data curation, formal analysis, methodology, software, writing-original draft, writing-review\&editing of the manuscript.

Email: tangjiqin0312@163.com

Author 2 - gong zhang - Author 2 is Gong Zhang, who is drafted the conceptualization, data curation, formal analysis, methodology, software, writingoriginal draft, writing-review\&editing of the manuscript. Also contributed to the development of the selection criteria and the risk of bias assessment strategy.

Email: zhangyoulin0905@163.com

Author 3 - jinxiao xing - Author 3 is Jinxiao Xing, who is drafted the conceptualization, data curation, formal analysis, methodology, software, writing-original draft, writing-review\&editing of the manuscript.

Email: xingjingxiao2021@163.com

Author 4 - ying yu - Author 4 is Ying Yu, who is drafted the project administration, supervision and revised the manuscript. Also provided important supports during design of the study and collection, analysis, and interpretation of data and in writing the manuscript.

Email: 13969179693@163.com

Author 5 - tao han - Author 5 is Tao Han, who is drafted the project administration, supervision and revised the manuscript. The funder(Tao Han) provided important supports during design of the study and collection, analysis, and interpretation of data and in writing the manuscript.

Email: 60012002@sdutcm.edu.cn 